\title{
Performance Evaluation of Job Allocation and Load Balancing Methods Using Meta-Heuristic Function in Public Cloud Network
}

\author{
Indra Nath Sahu ${ }^{1}$, Dr. Jitendra Sheetalani ${ }^{2}$ \\ ${ }^{1,2}$ (Sri Satya Sai University \& Medical Sciences, Sehore, India)
}

\begin{abstract}
Job allocation and balancing of load is major issue in public cloud network. The allocation of job and resource used queening principle, the queueing principle creates the job wanting condition and finally job allocation process is failed. For the improvement of job allocation process used meta-heuristic function and dynamic load balancing technique. the dynamic load balancing technique offers various methods for the job allocation and resource management. In this paper evaluate the performance of job allocation technique in cloud network based on different heuristic function such as genetic algorithm, ant colony optimization algorithm and particle swarm optimization. The heuristic function increases the performance of cloud network in interdisciplinary environments. The evaluation process used MATLAB software and design different scenario of load over public cloud network.
\end{abstract}

Keywords: Cloud Computing. Job Allocation, heuristic function, Load Design, MATLAB

\section{Introduction}

Public cloud network enhanced the middle and enterprise level business policy and market trends. Public cloud network offers various services over the internet based on rent based services. the limited number of resource and unlimited number of request over the internet raised the issue of network traffic and job failure and allocation problem is occurred. The failure of job and allocation process degraded the performance of public cloud network. For the enhancement of the performance used various load balancing and scheduling technique for the management of load over the public cloud network. The scheduling optimization is very significant because the scheduling is a main house block for making Cloud more available to user communities. The optimization methods for Cloud scheduling are the main objective for this dissertation[32][33][37]. The scheduling problem is just like NP-Complete. Therefore, approximation algorithms are careful, which are expected to quickly offer a solution, even if it is only near to-optimal. Computational cloud is a requirement for many Cloud and cloud computing applications. Computational cloud might refer to the completion of allocated task, minimization of execution time for task scheduling. In simple words, Cloud computing share and maintain geographically distributed resources allowing user to use computational capabilities and storage space for specific applications. It is a category of distributed computing which often dedicated to particular application or scientific problem that requires a great number of computer processing cycles or access to large amounts of data [17]. With these processing needs, Cloud share getting more attention towards the large-scale computation problems. The strategy of cloud is to use middleware to divide and apportion pieces of a program among several computers. Cloud computing involves computation in a distributed manner, which may also involve the aggregate of large-scale computers. This environment is a way to enhance the efficiency of underlying system by maximizing utilization of distributed resources. In large distributed systems such as cloud systems, there are often large amounts of resources available to be used for processing the jobs. Because of these computational resources can cost huge investment, so their maximum utilization has been taken an important consideration for cloud owners? Job scheduling plays an important role in the efficient management of resources on cloud computing environment[20][22]. To achieve better performance of Computational cloud, the resources must be utilized at maximum potential to reduce the idle time of it. The objective of cloud scheduling is to utilize the available resources in the distributed environment. For the allocation of Job and resource there is no any standard method for the execution of task. In normal case computational cloud used first come first allocation technique (FCFS)[34][35][36]. In the case of FCFS technique the failure of Job is very high and all cases the performance of cloud computing is degraded. Now a day's various authors used a heuristic and meta-heuristic function for the improvement of computational cloud. In consequence of these function such as genetic algorithm, hill calming algorithm and various algorithm are used. Some authors focus on the ant colony optimization technique for the allocation of job. If the job size is increase the allocation of job is faced a problem of selection method. The process of selection method used different constraints function for the selection of Jobs [18][19][21]. The rest of paper discuss as section II. Balancing policy in section III. Discuss related work. In IV discuss experimental result and finally discuss conclusion \& future work in section V. 


\section{Balancing Policy}

In cloud computing, the limited numbers of resource allocate the maximum consumer and handle maximum job profit. Due to this reason, the process of cloud scheduling is overloaded and the performance of cloud computing is decrease. For the improvement of job scheduling and allocation of job required the process of load balancing technique. Initially cloud load balancing technique divided into two sections one is static load balancing technique and other is dynamic load balancing technique. The static load balancing technique used CPU scheduling such as round robin, first come first served and another static algorithm. Now a days used dynamic algorithm such as genetic algorithm, ant colony optimization and many more algorithm based on heuristic function. Various authors and researcher used a concept of fuzzy logic and other classical set theory approach[23][24][27].

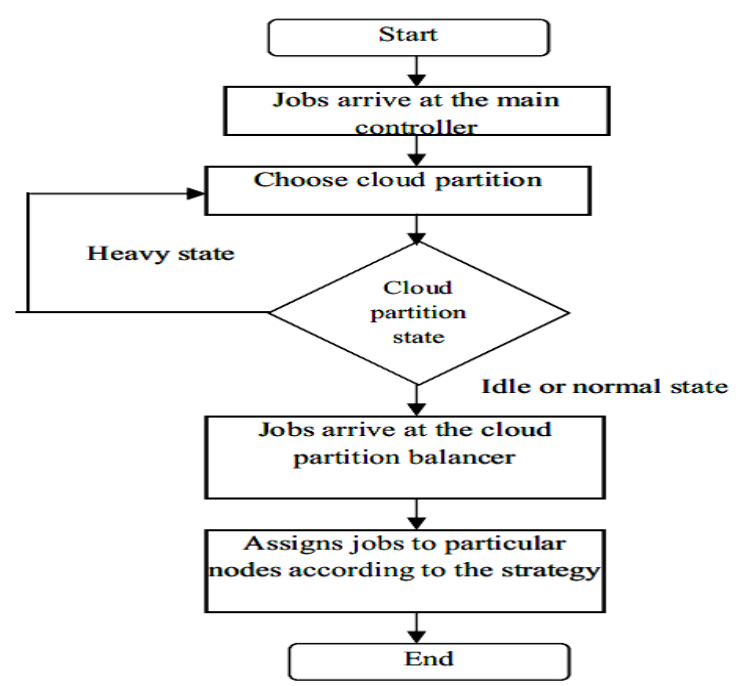

Fig. 1: job allocation processes in public cloud computing model

How to used different mode of load balancing algorithm for the process of job allocation and job selection. The process of categorization of algorithm depends on the process of work started and load balancing policy. The policy of load balancing divided into three section one is data sender, data receiver and combination of both[25][26][29].

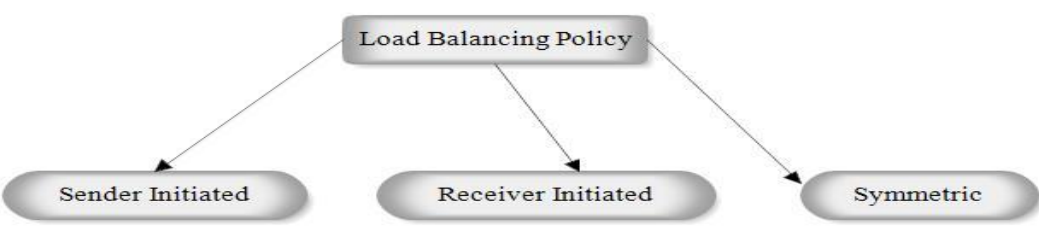

Fig. 2: shows that distribution of load balancing policy

The process of load balancing technique categorized into two sections one is static and other is dynamic. Static: the process of static load balancing technique work based on pervious information, its do not depends on the current state information. Dynamic: the concept of dynamic load balancing technique does not depend on the pervious information; it acts on the basis of current situation of process. Now a day's used four policies for load balancing technique these technique used transfer policy, location policy, selection policy and information policy[27][31][32].

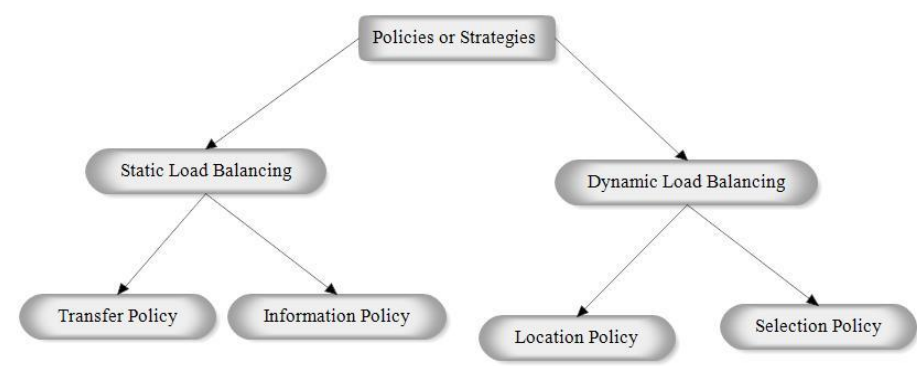

Fig. 3: shows that distribution of load balancing policy. 


\section{Related Work}

In this section discuss the related work in the fields of public cloud network for load balancing and allocation of job and resource. Kui Ren, Cong Wang and Qian Wang Et al. [1] as indicated by authors, Cloud registering speaks to today's most energizing processing outlook change in data innovation. The authors diagram a few basic security challenges and spur encourage examination of security answers for a dependable open cloud condition. they have talked about a few basic security challenges that momentum explore pushes aren't yet tending to. This article is proposed as a call for activity to inspire advance examination of the many testing security issues that will affect the general population cloud's future. Unmistakably, much work stays for a dependable open cloud condition to end up distinctly a reality. Yongjun Ren, Jian Shen, Jin Wang, Jin Han and Sungyoung Lee Et al. [2] in this paper, they examined a proficient shared unquestionable provable information ownership conspire, first they characterize MV-PDP framework model and security display. And after that they use of Diffie-Hellman shared key to build the homomorphic authenticator. In MV-PDP framework, the information squares marked by a customer can be confirmed by a private verifier, while the information pieces marked by a verifier can likewise be check by a customer. What's more, similar information pieces are anything but difficult to be marked and checked by a customer and the verifier thus. They jump at the chance to accentuate that ECC-based homomorphic authenticator is utilized to outline their plan, which result in low figuring and Communications because of the way that bilinear operation is not required. Wayne Jansen and Timothy Grance Et al. [3] they have talked about Cloud processing can and means distinctive things to various individuals. The normal attributes most understandings share is on-request versatility of very accessible and dependable pooled processing assets, secure access to metered administrations from almost anyplace, and dislodging of information and administrations from inside to outside the association. Cloud suppliers may choose to offer or consolidation their offerings with different organizations; benefit offerings might be overshadowed by those of another cloud supplier or fall into disapproval; and associations might be required to re-contend a current contract for cloud administrations, when all choice years have been depleted. In the end displacing a few frameworks to another open cloud is a particular plausibility that government offices and different associations must not ignore. Ang Li, Xiaowei Yang, Srikanth Kandula and Ming Zhang Et al. [4] authors acquainted with trust their approach is a huge stride toward empowering quick, precise supplier choice for a great many developing cloud applications. Be that as it may, there's still much to do around there. For instance, each cloud benefit merits its own particular careful, efficient execution examination. This can give knowledge in how to enhance the current applications to better fit the cloud condition. Besides, execution won't not be the main foundation for choosing a supplier. Perspectives, for example, oversee capacity, accessibility, and information excess likewise concern the clients. Instructions to incorporate these elements into a total, simple to-utilize supplier choice system remains an open test. Gaochao Xu, Junjie Pang and Xiaodong Et al. [5] This article presents a superior load adjust display for the general population cloud in light of the cloud apportioning idea with a change component to pick distinctive systems for various circumstances. The calculation applies the amusement hypothesis to the heap adjusting procedure to enhance the productivity in people in general cloud condition. In the information insights investigation, the fundamental controller and the cloud segment balancers need to revive the data at a settled period. In the event that the period is too short, the high recurrence will impact the framework execution. On the off chance that the period is too long, the data will be excessively old, making it impossible to settle on great choice. Along these lines, tests and factual instruments are expected to set a sensible revive periods. HuijunXiong, Xinwen Zhang, Danfeng Yao, Xiaoxin Wu and Yonggang Wen Et al. [6] CloudSeal flawlessly coordinates symmetric encryption, intermediary based re-encryption, k-out-of-n mystery sharing, and communicate repudiation instruments. These calculations permit CloudSeal to reserve the real piece of a put away figure content question in the conveyance organize for substance circulation, while keeping the minor part in the distributed storage for key administration. They planned and actualized CloudSeal, a conclusion to-end content confidentiality security instrument for huge scale content stockpiling and dissemination frameworks over people in general cloud foundation. By utilizing progressed cryptographic calculations including symmetric encryption, intermediary based re-encryption, limit mystery sharing, and communicate repudiation, CloudSeal addresses special difficulties of efficient figure content change, figure content reserving in the conveyance arrange, and adaptable client and key administration. Through the model actualized on Amazon EC2, S3, and CloudFront, their trial assessment exhibits that CloudSeal accomplishes the efficiency and maintains a strategic distance from conceivable execution bottleneck. Jiawei Yuan and Shucheng Yu Et al. [7] In this paper, various plans have been talked about in view of the ideas of POR, PDP, POW and POSD. In this work, they filled the crevice amongst POR and POW and talked about a consistent cost conspire that accomplishes secure open information respectability examining and capacity deduplication in the meantime. their examined plot empowers the deduplication of both records and their relating validation labels. The security of their PCAD plan is demonstrated in view of the CDH issue, the Static Diffie-Hellman issue and the t-SDH issue. they approve the productivity and adaptability of their plan through numerical examination and exploratory outcomes on Amazon EC2 Cloud. their examined polynomial based 
validation tag can likewise be utilized as a free answer for other related applications, for example, certain SQL seek, encoded watchword look, and so forth. Gabriele D'Angelo Et al. [8] they talked about another approach in view of multi-specialist frameworks for the reproduction of complex frameworks. It is conceivable to actualize propelled procedures, for example, the movement of reenacted substances keeping in mind the end goal to construct systems that are both versatile and simple to utilize. Versatile components can significantly decrease the correspondence cost in the parallel/dispersed models, to actualize stack adjust strategies and to adapt to execution situations that are both variable and element. At last, such systems will be utilized to assemble reproductions on top of untrustworthy cloud administrations. Its principle trademark is the versatile movement of the mimicked elements between the execution units. Systems in view of heuristics can be executed for both lessening the correspondence cost and load adjusting the recreation. their proposition has been actualized in the ART'IS/GAIA+ recreation middleware and tried with many models, and the got execution results are extremely encouraging. Lei Xu, Xiaoxin Wu and Xinwen Zhang Et al. [9] they examined CL-PRE, a certificateless intermediary re-encryption conspire for secure information offering to open cloud. In CL-PRE, an information proprietor encodes imparted information in cloud to an encryption key, which is further scrambled and changed by cloud, and after that conveyed to authentic beneficiaries as per get to control. Consider an intermediary running in broad daylight cloud to use flexible distributed storage and processing assets, they additionally talked about multi-intermediary CL-PRE-to send middle of the road intermediaries in various cloud specialist co-ops with a specific end goal to enhance the vigor of the framework, and randomized CL-PRE-to randomize the reencryption key each season of information partaking so as to decrease the trust on the intermediary. their execution assessments confirm that their examined plans are down to earth for cloud-based applications. Katrina LaCurts, Shuo Deng, Ameesh Goyal and Hari Balakrishnan Et al. [10] This paper demonstrates that occupants can make a superior showing with regards to setting applications by understanding the basic cloud organize and also the requests of the applications. To do as such, inhabitants must have the capacity to rapidly and precisely measure the cloud system and profile their applications, and after that utilization a system mindful position strategy to place applications. In this paper, they roused the requirement for system mindful application situation on distributed computing frameworks. As applications turn out to be more system serious, they can get to be bottlenecked by the system, even in very much provisioned mists. their tests on Amazon EC2 demonstrated that Choreo enhances application finishing time by a normal of $8 \%-14 \%$ when applications are put at the same time, and $22 \%-43 \%$ when they touch base progressively, contrasted with option situation plans contemplated on reasonable workloads. Joanna Kołodziej, Samee Ullah Khan, Lizhe Wang and Albert Y. Zomaya Et al. [11] In this paper, they looked at experimentally the adequacy of the talked about hereditary schedulers with three other well known heuristic framework schedulers, to be specific Min-Min, Relative cost and Tabu inquiry in three network measure situations and static and element cases. To give the trials, they incorporated all vitality mindful schedulers inside a matrix test system. The accomplished recreation comes about confirmed the adequacy of the examined GA-based schedulers in the lessening of the vitality devoured by the entire framework and in element stack adjusting of the assets in network groups that is sufficient to keep up the coveted quality level(s). The considered planning situation is exceptionally straightforward; truth be told, they just defined two criteria, and the streamlining of the Makespan prompts the change of the heap adjusting of the assets. The greater part of the practical booking issues in frameworks are profoundly parametrized. The introduced determination and reproduction model can be effectively reached out by actualizing multilevel hierarchic and cross breed hereditary schedulers and multi-populace hereditary metaheuristic. M. Abu Sharkh, M. Jammal, A. Shami and A. Ouda Et al. [12] Each supplier offers a one of a kind administration portfolio with a scope of asset arrangements. This paper presents some interior and outer variables that influence the outline of DC RA models. Outside difficulties are generally brought on by regulative, topographical and charging model related variables. Interior difficulties incorporate expanding the advantages from information territory highlights. They likewise incorporate planning a dependable inward DC organize. Other interior variables are identified with SDN, adaptation to internal failure and transportability. Outlining a vitality mindful RA display confronts execution challenges that are for the most part created by combination, VM relocation and server sit out of gear state design. These outline difficulties are talked about with the point of giving a reference to be utilized when planning a far reaching vitality mindful asset designation show for CC server farms. Qi Zhang, Quanyan Zhu and RaoufBoutaba Et al. [13] In this paper, they consider the instance of a solitary cloud supplier and deliver the question how to best match client request as far as both supply and cost with a specific end goal to amplify the supplier's income and client fulfillments while limiting vitality cost. To begin with, in this paper they have only concentrated an imposing business model market situation from an IaaS supplier's point of view. It is additionally vital to break down the framework from client's viewpoint as far as offering conduct and administration quality. Second, they might want to determine a more precise model for request reaction out in the open cloud condition. they are additionally inspired by leading more broad trials utilizing workload informational indexes that contain value data. Javier Espadas, Arturo Molina, Guillermo Jiménez, Martín Molina, Raúl Ramírez and David Concha Et al. [14] This model contains three corresponding methodologies: 
(1) inhabitant based confinement which embodies the execution of each occupant, (2) occupant based load adjusting which circulates demands as indicated by the occupant data, and (3) an occupant based VM occasion designation which decides the quantity of VM cases required for certain workload, in view of VM limit and occupant setting weight. Subsequent to running all tests and recreations, the outcomes were assembled and midpoints were figured. When all is said in done, over and underutilization midpoints were lessened however midpoints for underutilization were measurably progressed. M. Reza Rahimi, Nalini Venkatasubramanian and Athanasios V. Vasilakos Et al. [15] In this paper, they talked about a novel structure to model portable applications as an area time workflows (LTW) of errands; here client versatility examples are meant a portable administration use designs. They talked about another system to model versatile applications as a Location-Time Workflow - the one of a kind viewpoint here is that this deliberation models the portable client benefit use designs in light of client portability. their fundamental objective was to utilize this idea to ideally break down the arrangement of undertakings to execute on the versatile customers and the 2-level cloud design, considering various QoS variables like power, cost, and postponement. they talked about an efficient calculation called MuSIC that can accomplish around 78\% of ideal arrangements when the quantity of portable clients is high. their reviews likewise demonstrate that MuSIC performs great under vulnerability in expectation of portable client LTW and distinctive portability designs like arbitrary waypoint and Manhattan models. K. Shyamala and T. Sunitha Rani Et al. [16] This paper uncovers how successfully asset designation issue can be tended to in the viewpoint of cloud specialist co-op and furthermore gives a similar examination which causes in choosing parameters to meet the target work for enhancing the request to expand the benefit. From the above examination, it can be reasoned that productive asset designation can enhance cost, time and power utilization. It can likewise limit the underutilization of assets, adjust stack, ask for misfortune and renting cost. A productive asset allotment amortizes the organization overhead as well as screens the malevolent clients making harm the framework and checks them from expanding their own utility. This relative investigation helps in choosing parameters that need much fixation to meet the goal work. It additionally indicates how request and limit influence productive asset designation.

\section{Experimental Analysis}

In this section discuss the experimental result of different heuristic function such as ant colony optimization, genetic algorithm and particle swarm optimization. All heuristic function support dynamic load balancing policy and job allocation mechanism. For the simulation of algorithm used MATLAB software and creates different load scenario and policy. The performance parameter measure the job compilation and job failure in public cloud network [24][30].

\section{Job completion}

Job completion is one of the most important standard metrics used to measure the performance of fault tolerant systems [5]. Job completion is defined as:

$$
\text { job Completion }=\frac{n}{T}
$$

Where $n$ is the total number of jobs submitted and Tnis the total amount of time necessary to complete $n$ jobs. Job completion is used to measure the ability of the grid to accommodate jobs.

\section{Job Failure}

It is the percentage of the tendency of the selected grid resources to fail and is defined as:

$$
\text { failure }=\frac{\sum_{j=1}^{m} P f j}{m} \times 100 \%
$$

Where $m$ is the total number of grid resources and $P f j$ is the failure rate of resource $j$. the faulty behavior of the system can be expected.

Through this metric,

\section{Comparative result analysis and cloud size model is $6 * 6$}

Table 1: Comparison for small job for MC-ANT, ANT, PSO and GA methods

\begin{tabular}{|l|l|l|l|l|}
\hline METHOD & NO. OF JOB & NO. OF RESOURCE & JOB FAILURE & JOB COMPLETION \\
\hline MC-ACO & 10 & 5 & 6.166667 & 99.166667 \\
\hline ANT & 10 & 5 & 2.000000 & 91.166667 \\
\hline PSO & 20 & 10 & 5.000000 & 86.500000 \\
\hline GA & 20 & 10 & 4.500000 & 95.333333 \\
\hline MC-ACO & 30 & 15 & 5.000000 & 86.833333 \\
\hline ANT & 30 & 15 & 4.333333 & 96.166667 \\
\hline
\end{tabular}


Performance Evaluation of Job Allocation and Load Balancing Methods Using Meta-Heuristic

\begin{tabular}{|c|c|c|c|c|}
\hline PSO & 40 & 20 & 4.000000 & 83.000000 \\
\hline GA & 40 & 20 & 2.000000 & 90.000000 \\
\hline MC-ACO & 50 & 25 & 5.000000 & 85.833333 \\
\hline ANT & 50 & 25 & 3.000000 & 89.666667 \\
\hline PSO & 60 & 30 & 5.333333 & 87.333333 \\
\hline GA & 60 & 30 & 6.666667 & 95.666667 \\
\hline MC-ACO & 70 & 35 & 4.500000 & 84.833333 \\
\hline ANT & 70 & 35 & 3.000000 & 86.166667 \\
\hline PSO & 80 & 40 & 4.000000 & 83.500000 \\
\hline GA & 80 & 40 & 3.500000 & 86.000000 \\
\hline MC-ACO & 90 & 45 & 4.500000 & 82.333333 \\
\hline ANT & 90 & 45 & 3.000000 & 90.500000 \\
\hline PSO & 100 & 50 & 4.500000 & 82.333333 \\
\hline GA & 100 & 50 & 3.500000 & 87.333333 \\
\hline
\end{tabular}

Table 2: Comparison for middle job for MC-ANT, ANT, PSO and GA methods.

\begin{tabular}{|c|c|c|c|c|}
\hline METHOD & NO. OF JOB & NO. OF RESOURCE & JOB FAILURE & JOB COMPLETION \\
\hline MC-ACO & 100 & 50 & 4.000000 & 84.166667 \\
\hline ANT & 100 & 50 & 2.000000 & 95.000000 \\
\hline PSO & 200 & 100 & 4.500000 & 83.000000 \\
\hline GA & 200 & 100 & 3.000000 & 87.500000 \\
\hline MC-ACO & 300 & 150 & 4.500000 & 82.333333 \\
\hline ANT & 300 & 150 & 3.000000 & 88.500000 \\
\hline PSO & 400 & 200 & 4.000000 & 83.500000 \\
\hline GA & 400 & 200 & 4.833333 & 94.500000 \\
\hline MC-ACO & 500 & 250 & 5.000000 & 88.333333 \\
\hline ANT & 500 & 250 & 4.666667 & 91.166667 \\
\hline PSO & 600 & 300 & 4.500000 & 84.333333 \\
\hline GA & 600 & 300 & 7.166667 & 96.000000 \\
\hline MC-ACO & 700 & 350 & 4.500000 & 86.833333 \\
\hline ANT & 700 & 350 & 3.500000 & 87.333333 \\
\hline PSO & 800 & 400 & 7.333333 & 86.500000 \\
\hline GA & 800 & 400 & 4.666667 & 93.166667 \\
\hline MC-ACO & 900 & 450 & 6.166667 & 88.833333 \\
\hline ANT & 900 & 450 & 2.000000 & 95.000000 \\
\hline PSO & 1000 & 500 & 4.000000 & 83.500000 \\
\hline GA & 1000 & 500 & 3.000000 & 86.166667 \\
\hline
\end{tabular}

Table 3: Comparison for Large job for MC-ANT, ANT, PSO and GA methods.

\begin{tabular}{|c|c|c|c|c|}
\hline METHOD & NO. OF JOB & NO. OF RESOURCE & JOB FAILURE & JOB COMPLETION \\
\hline MC-ACO & 1000 & 500 & 4.000000 & 84.500000 \\
\hline ANT & 1000 & 500 & 3.000000 & 90.500000 \\
\hline PSO & 2000 & 1000 & 4.500000 & 83.000000 \\
\hline GA & 2000 & 1000 & 3.000000 & 89.666667 \\
\hline MC-ACO & 3000 & 1500 & 4.000000 & 83.500000 \\
\hline ANT & 3000 & 1500 & 3.000000 & 88.500000 \\
\hline PSO & 4000 & 2000 & 4.000000 & 84.500000 \\
\hline GA & 4000 & 2000 & 3.000000 & 90.500000 \\
\hline MC-ACO & 5000 & 2500 & 4.000000 & 82.333333 \\
\hline ANT & 5000 & 2500 & 3.000000 & 86.166667 \\
\hline PSO & 6000 & 3000 & 3.333333 & 87.500000 \\
\hline GA & 6000 & 3000 & 2.333333 & 94.833333 \\
\hline MC-ACO & 7000 & 3500 & 4.500000 & 83.500000 \\
\hline ANT & 7000 & 3500 & 3.500000 & 88.500000 \\
\hline PSO & 8000 & 4000 & 4.500000 & 83.000000 \\
\hline GA & 8000 & 4000 & 3.500000 & 87.333333 \\
\hline MC-ACO & 9000 & 4500 & 4.000000 & 84.666667 \\
\hline ANT & 9000 & 4500 & 3.000000 & 90.833333 \\
\hline PSO & 10000 & 5000 & 4.000000 & 84.500000 \\
\hline GA & 10000 & 5000 & 3.000000 & 90.500000 \\
\hline
\end{tabular}




\section{Result Analysis of Scheduling Method}

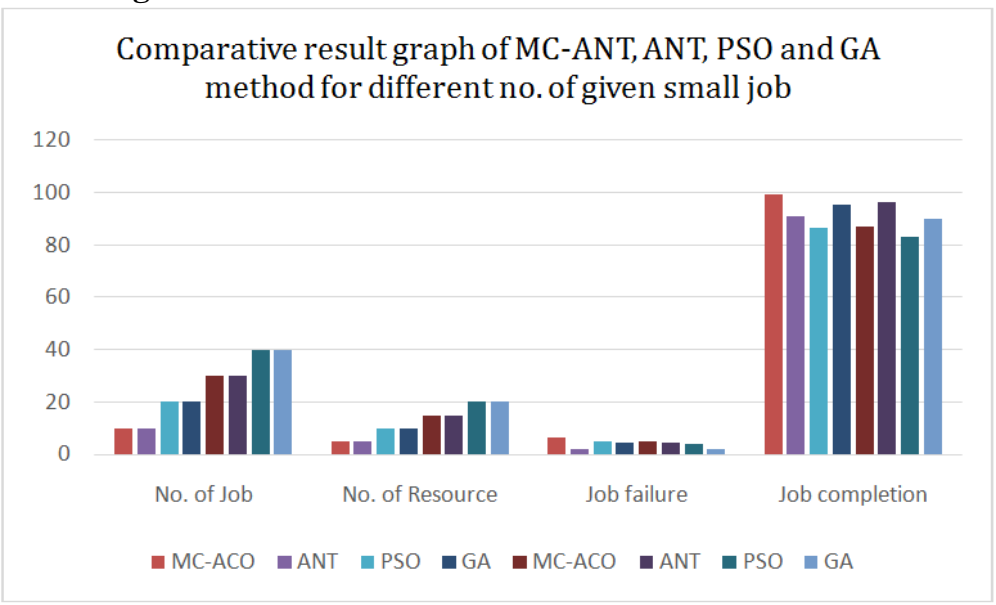

Fig.4: Comparative result analysis of Small job for all method of job scheduling

The graph result shows that MC-ANT, ANT, PSO and GA technique for job scheduling is better in comprassion of all method.

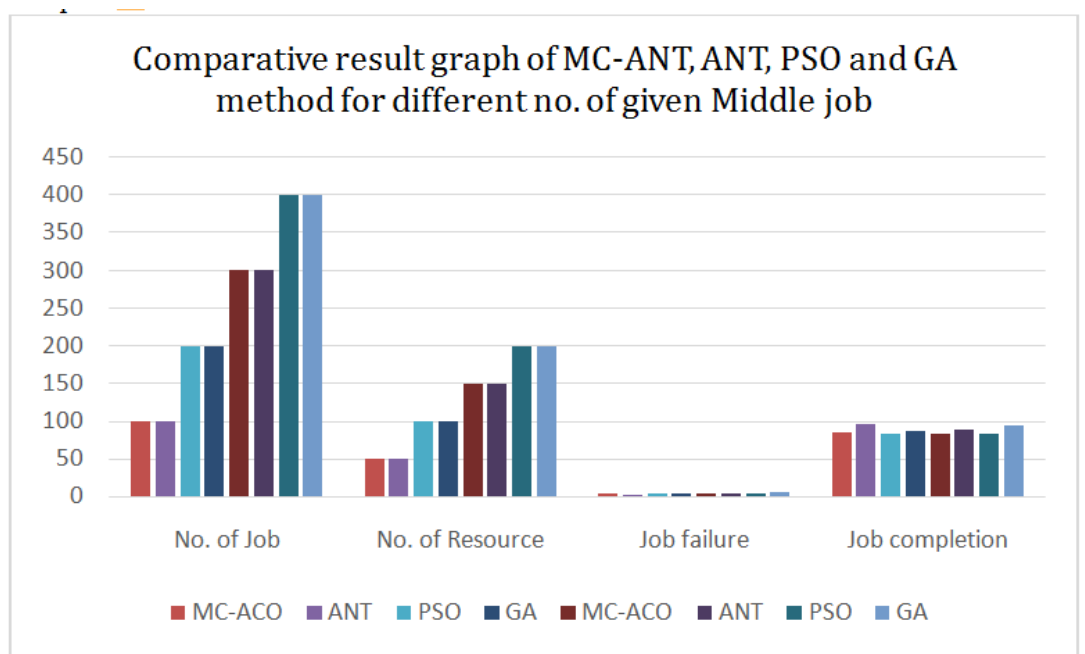

Fig.5: Comparative result analysis of Middle job for all method of job scheduling

The graph result shows that MC-ANT, ANT, PSO and GA technique for job scheduling is better in comprassion of all method.

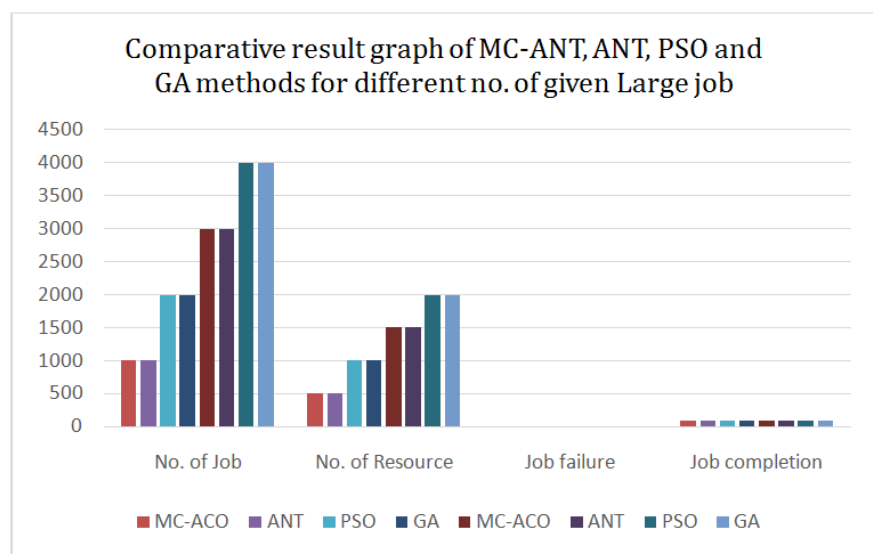

Fig.6: Comparative result analysis of Large job for all method of job scheduling

The graph result shows that MC-ANT, ANT, PSO and GA technique for job scheduling is better in comprassion of all method. 


\section{Conclusion \& Future Scope}

In this paper study of various job and resource allocation technique in public cloud network. The threemajor algorithm is considering here GA, ACO and PSO. The framework assignment planning calculation in view of the utilization of hereditary calculation and subterranean insect state calculation accomplishes preferred outcomes over the matrix undertaking booking calculation just based on insect province calculation. We expect this calculation is connected to the real undertaking of booking network, to acquire a more solid and powerful outcome instead of based on the model on the premise of from the earlier keeping in mind the end goal to test our proposed calculation, we shift the quantity of employments submitted to matrix. Matrix execution is outline as $10 * 10,20 * 5$ and $6 * 6$ planning issue. Expanding the effectiveness of computational lattice utilized instructor learning based enhancement system for choice of occupation in multi criteria subterranean insect province improvement calculation (MCACO) for framework planning. Some point examines in light of employment disappointment, work fruition and make traverse time. Overall examination of result gives the data that PSO work better in the event of little and medium size of network let. The ease of use and utilization of computational lattice are critical calculate current situation of dispersed processing and distributed computing. The handling heart of both advances is computational framework.

\section{References}

[1] Kui Ren, Cong Wang and Qian Wang, Security Challenges for the Public Cloud, IEEE, 2012, 69-73.

[2] Yongjun Ren, Jian Shen, Jin Wang, Jin Han and Sungyoung Lee, Mutual Verifiable Provable Data Auditing in Public Cloud Storage, Mutual Verifiable Provable Data Auditing in Public Cloud Storage, 2015, 317-323.

[3] Wayne Jansen and Timothy Grance, Guidelines on Security and Privacy in Public Cloud Computing, NIST, 2011, 1-70.

[4] Ang Li, Xiaowei Yang, Srikanth Kandula and Ming Zhang, Comparing Public- Cloud Providers, IEEE, 2011, 50-53.

[5] Gaochao Xu, Junjie Pang and Xiaodong, A Load Balancing Model Based on Cloud Partitioning for the Public Cloud, Tsinghua Science And Technology, 2013, 34-39.

[6] HuijunXiong, Xinwen Zhang, Danfeng Yao, Xiaoxin Wu and Yonggang Wen, Towards End-to-End Secure Content Storage and Delivery with Public Cloud, ACM, 2012, 1-10.

[7] Jiawei Yuan and Shucheng Yu, Secure and Constant Cost Public Cloud Storage Auditing with Deduplication, IEEE, 2013, 1-9.

[8] Gabriele D'Angelo, Parallel and Distributed Simulation from Many Cores to the Public Cloud (Extended Version), arXiv, 2014,1 9.

[9] Lei Xu, Xiaoxin Wu and Xinwen Zhang, CL-PRE: a Certificateless Proxy Re-Encryption Scheme for Secure Data Sharing with Public Cloud, ACM, 2012, 1-10.

[10] Katrina LaCurts, Shuo Deng, Ameesh Goyal and Hari Balakrishnan, Choreo: Network-Aware Task Placement for Cloud Applications, ACM, 2013, 1-14.

[11] Joanna Kołodziej, Samee Ullah Khan, Lizhe Wang and Albert Y. Zomaya, Energy efficient genetic-based schedulers in computational grids, John Wiley \& Sons, Ltd., 2012, 1-21.

[12] M. Abu Sharkh, M. Jammal, A. Shami and A. Ouda, Resource Allocation in a Network-Based Cloud Computing Environment: Design Challenges, IEEE, 2013, 1-7.

[13] Qi Zhang,Quanyan Zhu and RaoufBoutaba, Dynamic Resource Allocation for Spot Markets in Cloud Computing Environments, IEEE, 2011, 1-8.

[14] Javier Espadas, Arturo Molina, Guillermo Jiménez, Martín Molina, Raúl Ramírez and David Concha, A tenant-based resource allocation model for scaling Software-as-a-Service applications over cloud computing infrastructures, Future Generation Computer Systems, 2013, 273-286.

[15] M. Reza Rahimi, Nalini Venkatasubramanian and Athanasios V. Vasilakos, MuSIC: Mobility-Aware Optimal Service Allocation in Mobile Cloud Computing, 1-8.

[16] K. Shyamala and T. Sunitha Rani, An Analysis on Efficient Resource Allocation Mechanisms in Cloud Computing, Indian Journal of Science and Technology, 2015, 814-821.

[17] Mohammad Hammoud, M. Suhail Rehman and Majd F. Sakr, Center-of-Gravity Reduce Task Scheduling to Lower MapReduce Network Traffic, IEEE, 2013, 1-10.

[18] Keke Chen, James Powers,Shumin Guo and Fengguang Tian, CRESP: Towards Optimal Resource Provisioning for MapReduce Computing in Public Clouds, IEEE, 2014, 1-15.

[19] HadiGoudarzi and MassoudPedram, Multi-dimensional SLA-based Resource Allocation for Multi-Tier Cloud Computing Systems, IEEE, 2011, 1-8

[20] Balaji Palanisamy, Aameek Singh, Ling Liu and Bhushan Jain, Purlieus: Locality-aware Resource Allocation for MapReduce in a Cloud, ACM, 2011, 1-12.

[21] Edmund K. Burke, Matthew R. Hyde, Graham Kendall and John Woodward, Automating the Packing Heuristic Design Process with Genetic Programming, ACM, 2012, 1-25.

[22] Andrei Tchernykh, Uwe Schwiegelshohn, RaminYahyapour and Nikolai Kuzjurin, On-line hierarchical job scheduling on grids with admissible allocation, Springer, 2010, 545-552.

[23] Lu Huang, Hai-shan Chen and Ting-ting Hu, Survey on Resource Allocation Policy and Job Scheduling Algorithms of Cloud Computing, Journal of Software, 2013, 480-487.

[24] Jinn-Tsong Tsai Jia-Cen Fang and Jyh-Horng Chou, Optimized task scheduling and resource allocation on cloud computing environment using improved differential evolution algorithm, Elsevier, 2013, 3045-3055.

[25] Ruay-Shiung Chang and Chih-Yuan Lin, Chun-Fu Lin, An Adaptive Scoring Job Scheduling algorithm for grid computing, Information Sciences, 2012, 79-89.

[26] Raksha Sharma, Vishnu Kant Soni, Manoj Kumar Mishra and PrachetBhuyan, A Survey of Job Scheduling and Resource Management in Grid Computing, International Science Index, Computer and Information Engineering, 2010, 736-741.

[27] Jun $\mathrm{Wu}, \mathrm{Xin} \mathrm{Xu}$, Pengcheng Zhang and Chunming Liu, A novel multi-agent reinforcement learning approach for job scheduling in Grid computing, Future Generation Computer Systems, 2011, 430-439.

[28] FatosXhafa and Ajith Abraham, Computational models and heuristic methods for Grid scheduling problems, Future Generation Computer Systems, 2010, 608-621. 
[29] Mustafizur Rahman, Rafiul Hassan, Rajiv Ranjan and RajkumarBuyya, Adaptive workflow scheduling for dynamic grid and cloud computing environment, John Wiley \& Sons, Ltd., 2013, 1816-1842.

[30] SowmyaSuryadevera, Jaishri Chourasia, SonamRathore and Abdul Jhummarwala, Load Balancing in Computational Grids Using Ant Colony Optimization Algorithm, International Journal of Computer \& Communication Technology, 2012, 20-23.

[31] Jiayin Li, MeikangQiu, Jian-Wei Niu, Yu Chen and Zhong Ming, Adaptive Resource Allocation for Preemptable Jobs in Cloud Systems, IEEE, 2010, 31-36.

[32] Joanna Kołodziej and Samee Ullah Khan, Multi-level hierarchic genetic-based scheduling of independent jobs in dynamic heterogeneous grid environment, Elsevier, 2012, 1-19.

[33] Andrei Tchernykh, Johnatan E. Pecero, AritzBarrondo and Elisa Schaeffer, Adaptive energy efficient scheduling in Peer-to-Peer desktop grids, Future Generation Computer Systems, Elsevier, 2013, 1-12.

[34] MalarvizhiNandagopal and V. RhymendUthariaraj, Fault Tolerant Scheduling Strategy for Computational Grid Environment, International Journal of Engineering Science and Technology, 4361-4372.

[35] HesamIzakian, Behrouz TorkLadani, Ajith Abraham and V'aclavSnasel, A Discrete Particle Swarm Optimization Approach for Grid Job Scheduling, International Journal of Innovative Computing, Information and Control, 2010, 1-15.

[36] Saurabh Mandloi and Hitesh Gupta, Adaptive job Scheduling for Computational Grid based on Ant Colony Optimization with Genetic Parameter Selection, International Journal of Advanced Computer Research, 2013, 66-71.

[37] RikySubrata, Albert Y. Zomaya and Bjorn Landfeldt, Cooperative power-aware scheduling in grid computing environments, J. Parallel Distrib. Comput., 2010, 84-91. 